$11-1-2006$

\title{
Partial-Wave Expansions of Angular Spectra of Plane Waves
}

James A. Lock

Cleveland State University, j.lock@csuohio.edu

Follow this and additional works at: https://engagedscholarship.csuohio.edu/sciphysics_facpub

Part of the Physics Commons

How does access to this work benefit you? Let us know!

\section{Publisher's Statement}

This paper was published in Journal of the Optical Society of America A: Optics Image Science and Vision and is made available as an electronic reprint with the permission of OSA. The paper can be found at the following URL on the OSA website: http://www.opticsinfobase.org/josaa/ abstract.cfm?URI=josaa-23-11-2803. Systematic or multiple reproduction or distribution to multiple locations via electronic or other means is prohibited and is subject to penalties under law.

\section{Original Citation}

Lock, James A. "Partial-Wave Expansions of Angular Spectra of Plane Waves." Journal of the Optical Society of America A: Optics Image Science and Vision 23 (2006): 2803-2809.

\section{Repository Citation}

Lock, James A., "Partial-Wave Expansions of Angular Spectra of Plane Waves" (2006). Physics Faculty Publications. 93.

https://engagedscholarship.csuohio.edu/sciphysics_facpub/93

This Article is brought to you for free and open access by the Physics Department at EngagedScholarship@CSU. It has been accepted for inclusion in Physics Faculty Publications by an authorized administrator of EngagedScholarship@CSU. For more information, please contact library.es@csuohio.edu. 


\title{
Partial-wave expansions of angular spectra of plane waves
}

\author{
James A. Lock \\ Department of Physics, Cleveland State University, Cleveland, Ohio 44115
}

\begin{abstract}
Received March 16, 2006; revised May 22, 2006; accepted May 23, 2006; posted June 7, 2006 (Doc. ID 69019)
Focused electromagnetic beams are frequently modeled by either an angular spectrum of plane waves or a partial-wave sum of spherical multipole waves. The connection between these two beam models is explored here. The partial-wave expansion of an angular spectrum containing evanescent components is found to possess only odd partial waves. On the other hand, the partial-wave expansion of an alternate angular spectrum constructed so as to be free of evanescent components contains all partial waves but describes a propagating beam with a small amount of standing-wave component mixed in. A procedure is described for minimizing the standing-wave component so as to more accurately model a purely forward propagating experimental beam. (C) 2006 Optical Society of America

OCIS codes: $290.4020,260.1960$.
\end{abstract}

\section{INTRODUCTION}

When calculating electromagnetic scattering of a transversely localized beam, such as a Gaussian laser beam, by a spherical particle, one commonly models the beam by either (i) a weighted sum of monochromatic plane waves traveling in different directions called an angular spectrum of plane waves ${ }^{1-3}$ or (ii) a weighted sum of spherical multipole waves called a partial-wave sum. ${ }^{4-6}$ The purpose of this study is to examine the relationship between these two beam models. In particular, when attempting to describe the diffractive spreading of the beam's transverse profile as the beam propagates beyond its focal waist, one is often interested in a type of initial value problem. Namely, given the transverse profile in the beam's focal plane, one would like to uniquely determine either the weighting function of the plane waves in the angular spectrum or the beam shape coefficients of the spherical multipole waves in the partial-wave sum so as to determine the beam's amplitude everywhere in space. Once the angular spectrum weighting function or beam shape coefficients are known, scattering of the beam by a spherical particle may be calculated using standard methods. ${ }^{7}$ It has been shown that the beam shape coefficients can be determined if the beam amplitude is known either on the surface of a sphere ${ }^{5}$ of arbitrary radius $r$ or throughout all space. ${ }^{6}$ In these cases, one needs to know exactly how the beam evolves once it leaves its focal plane. The goal of this paper is to obtain the beam shape coefficients with more modest knowledge of the incident beam, namely, its amplitude only in the $z=0$ focal plane.

For the sake of simplicity, I examine a beam with a scalar amplitude rather than an electromagnetic beam whose electric and magnetic field amplitudes are vectors. However, by beginning the analysis of electromagnetic beams with the Bromwich scalar radiation potentials, ${ }^{6}$ the scalar wave results obtained here turn out to be applicable, with only minor modification, to electromagnetic waves as well. The beams considered here are assumed to impinge directly on the origin of coordinates and are called on-axis beams. The extension of these results to offaxis beams, which propagate past the origin with a nonzero impact parameter, will be considered elsewhere. Two different versions of the angular spectrum are examined. The first is obtained by integrating over the $k_{x}, k_{y}$ plane in Fourier transform space and contains only forwardpropagating components, though some of them are evanescent. ${ }^{1-3}$ The beam obtained using this angular spectrum is well suited for diffraction problems. But the presence of the evanescent components causes certain complications in the beam's partial-wave analysis. An alternate angular spectrum is obtained by integrating over a sphere of radius $k$ in Fourier transform space. It contains both forward and backward propagating components and is free of evanescent components. The beam described by this angular spectrum is dominantly forward propagating, but has a small amount of standing-wave character mixed in. This beam is well suited to partialwave expansions and generalized Lorentz-Mie scattering calculations. A procedure for minimizing the standingwave portion of the alternate angular spectrum is developed so as to allow it to more accurately model a purely forward propagating experimental focused laser beam. Since the two beam models are different, the beams obtained from each model using the identical focal plane profile differ as well. But in the paraxial limit, these differences become insignificant.

The body of this paper is organized as follows. In Section 2, some of the consequences of the first angular spectrum of plane waves are examined, its partial-wave expansion is determined, and the scattered and interior partial-wave amplitudes when such a beam is scattered by a homogeneous sphere centered on the origin of coordinates are calculated. In Section 3, the same calculations are performed for the beam defined by the alternative angular spectrum. In Section 4, the principal results of Sections 2 and 3 are summarized and discussed. 


\section{ANGULAR SPECTRUM OF PLANE WAVES WITH EVANESCENT COMPONENTS}

\section{A. Beam Propagator}

Consider the monochromatic scalar wave beam $\psi_{\text {beam }}(\mathbf{r}) \exp (-i \omega t)$ with the free-space wave number $k$ $=2 \pi / \lambda$. For simplicity, the beam amplitude is assumed to be circularly symmetric, its axis coincides with the $z$ axis of a rectangular coordinate system, and the beam propagates in the positive $z$ direction. The wave amplitude $\psi_{\text {beam }}(\mathbf{r})$ satisfies the scalar wave equation,

$$
\left(\nabla^{2}+k^{2}\right) \psi_{\text {beam }}(\mathbf{r})=0
$$

An elementary solution of Eq. (1) is the plane-wave $\exp (\mathrm{ik} \cdot \mathbf{r})$ where $\mathbf{k}$ is the wave vector denoting both the plane wave's propagation direction and the wavelength, and

$$
\mathbf{r}=x \mathbf{u}_{x}+y \mathbf{u}_{y}+z \mathbf{u}_{z}
$$

are the rectangular coordinates of a point in space with respect to the origin. For future reference, the spherical coordinates of the point $\mathbf{r}$ are $(r, \theta, \phi)$. The monochromatic beam amplitude $\psi_{\text {beam }}(\mathbf{r})$ may be written as a weighted sum of plane waves integrated over the $k_{x}, k_{y}$ plane in Fourier transform space, ${ }^{1-3}$

$$
\begin{aligned}
\psi_{\text {beam }}(x, y, z)= & \left(1 / 4 \pi^{2}\right) \int_{-\infty}^{\infty} \mathrm{d} k_{x} \int_{-\infty}^{\infty} \mathrm{d} k_{y} A\left(k_{x}, k_{y}\right) \\
& \times \exp \left[i\left(k_{x} x+k_{y} y\right)\right] \exp \left[i\left(k^{2}-k_{x}^{2}-k_{y}^{2}\right)^{1 / 2} z\right] .
\end{aligned}
$$

Equation (3) is called an angular spectrum of plane waves. All the plane waves in the angular spectrum have a positive $z$ component and thus generally propagate in the forward direction. But the waves in the spectrum for which $k_{x}^{2}+k_{y}^{2}>k^{2}$ are evanescent and thus influence beam propagation only in the near field of the beam's $z=0$ focal plane. The weighting function $A\left(k_{x}, k_{y}\right)$ in Eq. (3) is commonly assumed to be sufficiently sharply peaked about $k_{x}=k_{y}=0$ so that the contribution provided by the evanescent waves can be ignored. In this section, some of the implications of retaining the evanescent waves are investigated.

In particular, I consider the initial value problem for which the beam amplitude at all points in space $\psi_{\text {beam }}(\mathbf{r})$ is to be determined from its amplitude in the $z=0$ plane, which I denote by $\psi_{\text {beam }}(\rho, 0)$ where $\rho$ is the radial coordinate in this plane. Taking the Fourier transform of Eq. (3) when $z=0$, one obtains

$$
A\left(k_{x}, k_{y}\right)=\int_{-\infty}^{\infty} \mathrm{d} x \int_{-\infty}^{\infty} \mathrm{d} y \psi_{\text {beam }}(x, y, 0) \exp \left[-i\left(k_{x} x+k_{y} y\right)\right] .
$$

If the beam is circularly symmetric, one may express the weighting function $A$ in terms of the polar coordinates $\rho_{k}$ and $\phi_{k}$ in the Fourier domain as

$$
A\left(\rho_{k}\right)=2 \pi \int_{0}^{\infty} \rho \mathrm{d} \rho \psi_{\text {beam }}(\rho, 0) J_{0}\left(\rho \rho_{k}\right)
$$

Substitution of Eq. (4) or Eq. (5) into Eq. (3) gives the beam at all points in space in terms of its amplitude in the $z=0$ plane.

Expanding further on this idea, let

$$
\mathbf{r}_{a}=x_{a} \mathbf{u}_{x}+y_{a} \mathbf{u}_{y}
$$

be the rectangular coordinates of a point in the $z=0$ plane with respect to the origin. For future reference, the spherical coordinates of $\mathbf{r}_{a}$ with respect to the origin are $\left(r_{a}, \theta_{a}, \phi_{a}\right)$ with $\theta_{a}=\pi / 2$. Similarly, let

$$
\mathbf{r}^{\prime}=\mathbf{r}-\mathbf{r}_{a}=x^{\prime} \mathbf{u}_{x}+y^{\prime} \mathbf{u}_{y}+z^{\prime} \mathbf{u}_{z}
$$

be the rectangular coordinates of the point $\mathbf{r}$ as observed in the translated coordinate system centered on $\mathbf{r}_{a}$. Again, for future reference the coordinates of $\mathbf{r}^{\prime}$ with respect to $\mathbf{r}_{a}$ in cylindrical and spherical coordinates are $\left(\rho^{\prime}, \phi^{\prime}, z^{\prime}\right)$ and $\left(r^{\prime}, \theta^{\prime}, \phi^{\prime}\right)$, respectively. One can then straightforwardly show that $\psi_{\text {beam }}(\mathbf{r})$ is the convolution of $\psi_{\text {beam }}\left(\mathbf{r}_{a}\right)$ with the so-called beam propagator $p\left(\mathbf{r}^{\prime}\right)$, i.e.,

$$
\begin{aligned}
\psi_{\text {beam }}(x, y, z)= & \int_{-\infty}^{\infty} \mathrm{d} x_{a} \int_{-\infty}^{\infty} \mathrm{d} y_{a} p\left(x-x_{a}, y\right. \\
& \left.-y_{a}, z\right) \psi_{\text {beam }}\left(x_{a}, y_{a}, 0\right)
\end{aligned}
$$

where

$$
\begin{aligned}
p\left(x^{\prime}, y^{\prime}, z^{\prime}\right)= & \left(1 / 4 \pi^{2}\right) \int_{-\infty}^{\infty} \mathrm{d} k_{x} \int_{-\infty}^{\infty} \mathrm{d} k_{y} \exp \left[\mathrm { i } \left(k_{x} x^{\prime}\right.\right. \\
& \left.\left.+k_{y} y^{\prime}\right)\right] \exp \left[\mathrm{i}\left(k^{2}-k_{x}^{2}-k_{y}^{2}\right)^{1 / 2} z^{\prime}\right] .
\end{aligned}
$$

The propagator of Eq. (9) is so named ${ }^{8,9}$ because it describes the propagation of the wave from its point of origin $\left(x_{a}, y_{a}, 0\right)$ to another point in space $(x, y, z)$. The beam propagator may be analytically evaluated as follows. For $z^{\prime}=0$, one immediately obtains

$$
p\left(x^{\prime}, y^{\prime}, 0\right)=\delta\left(x^{\prime}\right) \delta\left(y^{\prime}\right) .
$$

For $z \neq 0$, the integral in Eq. (9) may be evaluated analytically when $\mathbf{r}^{\prime}$ is expressed cylindrical coordinates and the integration in Fourier transform space is performed in polar coordinates,

$$
\begin{aligned}
p\left(\rho^{\prime}, \phi^{\prime}, z^{\prime}\right) & =(1 / 2 \pi) \int_{0}^{\infty} \rho_{k} \mathrm{~d} \rho_{k} J_{0}\left(\rho^{\prime} \rho_{k}\right) \exp \left[\mathrm{i}\left(k^{2}-\rho_{k}^{2}\right)^{1 / 2} z^{\prime}\right] \\
& =(1 / 2 \pi) \int_{0}^{k} w \mathrm{~d} w J_{0}\left[\rho^{\prime}\left(k^{2}-w^{2}\right)^{1 / 2}\right] \exp (\mathrm{i} z w) \\
& +(1 / 2 \pi) \int_{0}^{\infty} w \mathrm{~d} w J_{0}\left[\rho^{\prime}\left(k^{2}+w^{2}\right)^{1 / 2}\right] \exp (-z w) .
\end{aligned}
$$

The imaginary part of Eq. (11b) is free of evanescent components and is analytically known. ${ }^{10}$ The real part, containing both traveling and evanescent components, is also 
analytically known for $\rho^{\prime}=0$. For $\rho^{\prime} \neq 0$, the real part may be evaluated by expanding the integrand in powers of $\rho^{\prime}$ and integrating term by term. The final result is

$$
p\left(r^{\prime}, \theta^{\prime}, \phi^{\prime}\right)=\left(i k^{2} / 2 \pi\right) h_{1}^{(1)}\left(k r^{\prime}\right) P_{1}\left(\cos \theta^{\prime}\right),
$$

where

$$
\begin{aligned}
r^{\prime} & =\left(\rho^{\prime 2}+z^{\prime 2}\right)^{1 / 2}, \\
\cos \theta^{\prime} & =z^{\prime} / r^{\prime} .
\end{aligned}
$$

A similar calculation for $z^{\prime}<0$ gives

$$
p\left(r^{\prime}, \theta^{\prime}, \phi^{\prime}\right)=\left(\mathrm{i} k^{2} / 2 \pi\right) h_{1}^{(2)}\left(k r^{\prime}\right) P_{1}\left(\cos \theta^{\prime}\right) .
$$

This result warrants a number of comments. The factor $P_{1}\left(\cos \theta^{\prime}\right)$ in Eqs. (12a) and (13) ensures that the propagator vanishes everywhere in the $z=0$ plane except at the point $\mathbf{r}^{\prime}=0$ so as to avoid a discontinuity when crossing the $z=0$ plane and switching from the radially incoming wave $h_{1}^{(2)}\left(k r^{\prime}\right)$ to the radially outgoing wave $h_{1}^{(1)}\left(k r^{\prime}\right)$. Equation (12a) corresponds to the single spherical multipole wave or partial-wave $n^{\prime}=1, m^{\prime}=0$, which is the simplest spherical wave that avoids this discontinuity. Equation (12a) is also the Rayleigh-Sommerfeld propagator for the diffraction of scalar waves, ${ }^{11}$ and Eq. (8) is an expression of the Huygens mechanism of diffraction, namely, it is as if every point $\mathbf{r}_{a}$ in the $z=0$ plane is an effective point source of an outgoing $n^{\prime}=1, m^{\prime}=0$ spherical multipole wave given by Eq. (12a). The wave amplitude downstream from the $z=0$ plane is obtained by weighting each effective point source by the wave amplitude there and then adding together the waves from all the effective sources.

\section{B. Partial-Wave Analysis of the Angular Spectrum Beam}

To express Eq. (3) as a sum of partial waves centered on the origin instead of partial waves centered on $\mathbf{r}_{a}$, each outgoing wave of Eq. (12a) as a function of the spherical coordinates $\left(r^{\prime}, \theta^{\prime}, \phi^{\prime}\right)$ with respect to $\mathbf{r}_{a}$ must be reexpressed in terms of the spherical coordinates $(r, \theta, \phi)$ with respect to the origin. This is accomplished using the spherical multipole scalar wave translation formulas of Ref. 12 , in which the single outgoing spherical wave $n^{\prime}$ $=1, m^{\prime}=0$ centered on $\mathbf{r}_{a}$ becomes a linear combination of all the $n, m$ spherical multipole waves centered on the origin,

$$
\begin{aligned}
h_{1}^{(1)}\left(k r^{\prime}\right) P_{1}\left(\cos \theta^{\prime}\right)= & v \sum_{n, m}[(n-m) ! /(n+m-1) !] \\
& \times\left\{[(n-m+1) /(n+m)] j_{n}\left(k r_{<}\right) h_{n+1}^{(1)}\right. \\
& \times\left(k r_{>}\right) P_{n}^{m}\left(\cos \theta_{<}\right) P_{n+1}^{m}\left(\cos \theta_{>}\right) \\
& \times \exp \left(\mathrm{i} m \phi_{<}\right) \exp \left(-\mathrm{i} m \phi_{>}\right)-j_{n}\left(k r_{<}\right) h_{n-1}^{(1)} \\
& \times\left(k r_{>}\right) P_{n}^{m}\left(\cos \theta_{<}\right) P_{n-1}^{m}\left(\cos \theta_{>}\right) \\
& \left.\times \exp \left(\mathrm{i} m \phi_{<}\right) \exp \left(-\mathrm{i} m \phi_{>}\right)\right\},
\end{aligned}
$$

$$
\begin{aligned}
\left(r_{>}, \theta_{>}, \phi_{>}\right) & =\left\{\begin{array}{ll}
(r, \theta, \phi), & \text { if } r>a \\
\left(r_{a}, \theta_{a}, \phi_{a}\right), & \text { if } r<a
\end{array},\right. \\
\left(r_{<}, \theta_{<}, \phi_{<}\right) & =\left\{\begin{array}{ll}
\left(r_{a}, \theta_{a}, \phi_{a}\right), & \text { if } r>a \\
(r, \theta, \phi), & \text { if } r<a
\end{array},\right. \\
v & =\left\{\begin{array}{ll}
1, & \text { if } r>a \\
-1, & \text { if } r<a
\end{array} .\right.
\end{aligned}
$$

In Eqs. (14b) and (14c) $\theta_{>}$and $\theta_{<}$are not the greater and smaller angles but are the angles associated with the greater and smaller of the lengths $r$ and $a$. When Eq. (14a) is integrated over $\phi_{a}$ with $\theta_{a}=\pi / 2$, and assuming that $\psi_{\text {beam }}$ is circularly symmetric, only the $n=$ odd, $m=0$ spherical multipole waves remain and

$$
\begin{aligned}
\psi_{\text {beam }}(r, \theta, \phi)= & \sum_{n=\text { odd }} \mathrm{i}^{n}(2 n+1)[(n) ! ! /(n-1) ! !]\left[h_{n}^{(1)}\right. \\
& \times(k r) P_{n}(\cos \theta) \int_{0}^{r} k \mathrm{~d} \rho_{a} j_{n}\left(k \rho_{a}\right) \psi_{\text {beam }}\left(\rho_{a}, 0\right) \\
& +j_{n}(k r) P_{n}(\cos \theta) \int_{r}^{\infty} k \mathrm{~d} \rho_{a} h_{n}^{(1)} \\
& \left.\times\left(k \rho_{a}\right) \psi_{\text {beam }}\left(\rho_{a}, 0\right)\right] .
\end{aligned}
$$

The fact that only odd partial waves contribute to Eq. (15) is a consequence of the fact that the propagator of Eq. (12a) vanishes when $\theta=\pi / 2$. Finally, using ${ }^{14,15}$

$$
\int_{0}^{\infty} k \mathrm{~d} \rho_{a} j_{n}\left(k \rho_{a}\right)=\left\{\begin{array}{ll}
(n-1) ! ! /(n) ! !, & \text { if } n=\text { odd } \\
(\pi / 2)(n-1) ! ! /(n) ! !, & \text { if } n=\text { even }
\end{array},\right.
$$

the partial-wave expansion of the angular spectrum of plane waves of Eq. (3) for $z>0$ is

$$
\begin{aligned}
\psi_{\text {beam }}(r, \theta, \phi)= & \sum_{n \text { odd }} i^{n}(2 n+1)\left[\alpha_{n} h_{n}^{(1)}(k r)+i \beta_{n}(k r) j_{n}(k r)\right. \\
& \left.-i \gamma_{n}(k r) n_{n}(k r)\right] P_{n}(\cos \theta)
\end{aligned}
$$

where

$$
\alpha_{n}=\int_{0}^{\infty} k \mathrm{~d} \rho_{a} j_{n}\left(k \rho_{a}\right) \psi_{\text {beam }}\left(\rho_{a}, 0\right) / \int_{0}^{\infty} k \mathrm{~d} \rho_{a} j_{n}\left(k \rho_{a}\right),
$$

$$
\beta_{n}(k r)=\int_{r}^{\infty} k \mathrm{~d} \rho_{a} n_{n}\left(k \rho_{a}\right) \psi_{\text {beam }}\left(\rho_{a}, 0\right) / \int_{0}^{\infty} k \mathrm{~d} \rho_{a} j_{n}\left(k \rho_{a}\right),
$$

where ${ }^{13}$ 


$$
\gamma_{n}(k r)=\int_{r}^{\infty} k \mathrm{~d} \rho_{a} j_{n}\left(k \rho_{a}\right) \psi_{\text {beam }}\left(\rho_{a}, 0\right) / \int_{0}^{\infty} k \mathrm{~d} \rho_{a} j_{n}\left(k \rho_{a}\right) .
$$

For $z<0, \psi_{\text {beam }}(r, \theta, \phi)$ has the same form as Eq. (17a) except that the $\beta_{n}$ and $\gamma_{n}$ terms are opposite in sign and the radially incoming spherical Hankel function $h_{n}^{(2)}(k r)$ is used instead of $h_{n}^{(1)}(k r)$.

The partial-wave expansion of Eqs. (17a)-(17d) again warrants a number of comments. What will end up being the primary partial-wave amplitude, $\alpha_{n}$, is the normalized integral of the wave's amplitude in the $z=0$ plane weighted by the spherical Bessel function $j_{n}\left(k \rho_{a}\right)$. This integral separates out the $n$th partial-wave portion of $\psi_{\text {beam }}\left(\rho_{a}, 0\right)$. Further, since an outgoing spherical wave is being decomposed into partial waves, the presence of the radially outgoing Hankel function $h_{n}^{(1)}(k r)$ multiplying the beam shape coefficient $\alpha_{n}$ is not unexpected. Starting the integration interval in the numerator of Eq. (17c) at $\rho_{a}$ $=r$ avoids the divergence of the spherical Neumann function at $\rho_{a}=0$. The $\beta_{n}$ and $\gamma_{n}$ terms of Eq. (17a) are purely real and vanish as $r \rightarrow \infty$, thus influencing the evolution of the beam only in the near-zone region beyond $z=0$. It is thus tempting to associate these terms with the evanescent waves in Eq. (3), which are also purely real and also damp out as $r \rightarrow \infty$.

In the sharp peaking or paraxial approximation, $\psi_{\text {beam }}\left(\rho_{a}, 0\right)$ is sizable only for $\rho_{a} \lesssim w_{0}$ where $w_{0}$ is the beam's focal plane half-width, with the beam amplitude rapidly damping out for $\rho_{a}>w_{0}$. In this case, $\beta_{n} \approx \gamma_{n} \approx 0$ in the far-zone limit $r \gg w_{0}$ and the $\beta_{n}$ and $\gamma_{n}$ terms in Eq. (17a) may be neglected, leaving only the $\alpha_{n}$ term as the partial-wave sum. In addition, if we assume the beam width in its focal plane is large with respect to $\lambda$, then

$$
s \equiv 1 / k w_{0} \ll 1
$$

Using the size-bandwidth theorem, one then obtains

$$
1 / w_{0} \approx \rho_{k} \ll k
$$

and the evanescent components of the angular spectrum can be safely neglected. This same neglect is valid in diffraction situations where a plane wave is incident on an aperture of half-width $w_{0}$ much larger than the wavelength. Last, the paraxial approximation to the beam propagator can be obtained by Taylor series expanding $\left(k^{2}-k_{x}^{2}-k_{y}^{2}\right)^{1 / 2}$ in Eq. (9) and then performing the integrations over $k_{x}$ and $k_{y}$. For $z>0$ one obtains

$$
p(x, y, z)=(-i k / 2 \pi z) \exp (i k z) \exp \left[i k\left(x^{2}+y^{2}\right) / 2 z\right]
$$

which is the Fresnel diffraction propagator for scalar waves. Equivalently, the paraxial approximation can be thought of as modeling the beam as being slightly nonmonochromatic with

$$
k_{x}^{2}+k_{y}^{2}+k_{z}^{2} \approx k^{2}+\left(k_{x}^{2}+k_{y}^{2}\right)^{2} / 4 k^{2} .
$$

\section{Scattering of a Sphere by the Angular Spectrum} Beam

Scattering of the beam of Eq. (17a) by a sphere of radius $a$ and refractive index $N$ exhibits a number of interesting and novel properties. Defining

$$
\begin{aligned}
\xi_{n}(k r)= & \alpha_{n}-\gamma_{n}(k r) \\
& =\int_{0}^{r} k \mathrm{~d} \rho_{a} j_{n}\left(k \rho_{a}\right) \psi_{\text {beam }}\left(\rho_{a}, 0\right) / \int_{0}^{\infty} k \mathrm{~d} \rho_{a} j_{n}\left(k \rho_{a}\right), \\
S(\theta) & =\left\{\begin{array}{ll}
1, & \text { for } 0 \leqslant \theta<\pi / 2 \\
-1, & \text { for } \pi / 2<\theta \leqslant \pi
\end{array},\right. \\
\epsilon_{n}= & \begin{cases}1, & \text { for } n=\text { odd } \\
0, & \text { for } n=\text { even }\end{cases}
\end{aligned}
$$

Eq. (17a) and its extension to $z<0$ may be combined into the single expression,

$$
\begin{aligned}
\psi_{\text {beam }}(r, \theta, \phi)= & \sum_{n=0}^{\infty} \epsilon_{n} i^{n}(2 n+1)\left\{\alpha_{n} j_{n}(k r) P_{n}(\cos \theta)\right. \\
& +i\left[\beta_{n}(k r) j_{n}(k r)\right. \\
& \left.\left.+\xi_{n}(k r) n_{n}(k r)\right] S(\theta) P_{n}(\cos \theta)\right\} .
\end{aligned}
$$

The scattered wave for $r \geqslant a$ and the interior wave for $r$ $\leqslant a$ are assumed to be of the form ${ }^{7}$

$$
\psi_{\text {scatt }}(r, \theta, \phi)=-\sum_{n=0}^{\infty} i^{n}(2 n+1) b_{n} h_{n}^{(1)}(k r) P_{n}(\cos \theta),
$$

where $b_{n}$ are the partial-wave scattering amplitudes, and

$$
\psi_{\text {interior }}(r, \theta, \phi)=\sum_{n=0}^{\infty} i^{n}(2 n+1) d_{n} j_{n}(N k r) P_{n}(\cos \theta),
$$

where $d_{n}$ are the partial-wave interior amplitudes. Then the continuity of the wave amplitude and its radial derivative at $r=a$ permit the evaluation of $b_{n}$ and $d_{n}$. One obtains

$$
\begin{aligned}
b_{n}= & \epsilon_{n} \alpha_{n} M_{n} /\left(M_{n}+i D_{n}\right)+\sum_{p=0}^{\infty} \epsilon_{p}(p+1 / 2) i^{p+1-n} I_{n p} \\
& \times\left[\beta_{p}(k a) M_{p}+\xi_{p}(k a) D_{p}\right] /\left(M_{n}+i D_{n}\right), \\
d_{n}= & \epsilon_{n} \alpha_{n}\left[-i /(k a)^{2}\right] /\left(M_{n}+i D_{n}\right)+\sum_{p=0}^{\infty} \epsilon_{p}(p+1 / 2) i^{p+1-n} I_{n p} \\
& \times\left\{\left[-i \beta_{p}(k a)+\xi_{p}(k a)\right] /(k a)^{2}\right\} /\left(M_{n}+i D_{n}\right),
\end{aligned}
$$

where

$$
\begin{aligned}
& M_{n}=N j_{n}(k a) j_{n}^{\prime}(N k a)-j_{n}^{\prime}(k a) j_{n}(N k a), \\
& D_{n}=N n_{n}(k a) j_{n}^{\prime}(N k a)-n_{n}^{\prime}(k a) j_{n}(N k a), \\
& I_{n p}=\int_{0}^{\pi} \sin \theta \mathrm{d} \theta S(\theta) P_{n}(\cos \theta) P_{p}(\cos \theta) .
\end{aligned}
$$


Equations (26a) and (26b) have an interesting physical interpretation. The first term in $b_{n}$ and $d_{n}$ is the usual expression for scattering of a shaped beam by a sphere, i.e., the scattering and interior amplitudes for plane-wave incidence are multiplied by the beam shape coefficients $\alpha_{n}$. Since the incident beam contains only odd partial waves, the first term of Eqs. (26a) and (26b) applies to only odd partial waves as well. This term produces no scattering at $\theta=\pi / 2$ since $P_{n}(0)=0$ for odd $n$. Scattering at $\theta=\pi / 2$ can be produced only by even partial waves. The even partial waves in the scattering amplitude of Eq. (26a) are induced by the odd partial waves in the beam via the integral $I_{n p}$ in Eq. (28). This can be seen in the following way. When $S(\theta)$, which is an odd function with respect to $\theta$ $=\pi / 2$, is expanded in terms of the Legendre polynomials $P_{q}(\cos \theta)$ only the odd $q$ contribute. Equation (28) is then proportional to the integral of three Legendre polynomials, which is in turn proportional to the square of a Wigner $3-j$ symbol, ${ }^{16}$ coupling the angular momenta $n, p$, and $q$. Since $q$ and the incident partial waves $p$ are odd, the Wigner $3-j$ symbol is nonzero only for even scattered partial waves $n$, thus coupling odd partial waves in the incident beam to even partial waves of the scattered and interior fields. This coupling of different partial waves does not occur for scattering of a plane wave by a sphere, where each scattered partial wave is coupled to only the identical partial wave of the incident beam. Coupling of different partial waves also occurs in scattering by particles possessing less symmetry than that of a sphere. For example, for scattering of a plane wave by a spheroid or a sphere with surface roughness, each scattered partial wave is coupled to many incident beam partial waves via the Fourier decomposition of the particle's irregular shape. ${ }^{17}$ In Eqs. (26a) and (26b) on the other hand, each scattered partial wave is coupled to many incident beam partial waves by a combination of the beam's evanescent components and by the fact that a portion of the beam amplitude is proportional to the radially incoming wave $h_{n}^{(2)}(k r)$ as the beam approaches the sphere and is proportional to the radially outgoing wave $h_{n}^{(1)}(k r)$ after it leaves it. The coupled partial-wave scattering amplitudes $M_{p} /\left(M_{n}+i D_{n}\right)$ and $D_{p} /\left(M_{n}+i D_{n}\right)$ depend on both the odd partial wave $p$ of the incident beam and the even partial wave $n$ of the scattered field.

\section{ALTERNATE ANGULAR SPECTRUM OF PLANE WAVES}

A. Partial-Wave Analysis and Beam Shape Coefficients Another possibility for constructing an angular spectrum of plane waves is integrating the weighting function $A$ over a sphere of radius $k$ in Fourier transform space,

$$
\begin{aligned}
\psi_{\text {beam }}(r, \theta, \phi)= & (1 / 4 \pi) \int_{0}^{\pi} \sin \theta_{k} \mathrm{~d} \theta_{k} \\
& \times \int_{0}^{2 \pi} \mathrm{d} \phi_{k} A\left(\theta_{k}, \phi_{k}\right) \exp (\mathrm{ik} \cdot \mathbf{r}),
\end{aligned}
$$

rather than integrating over the $k_{x}, k_{y}$ plane as in Eq. (3). The beam of Eq. (29) is free of the evanescent components that complicated the analysis in Section 2. But now the presence of plane-wave components for both $0 \leqslant \theta_{k}<\pi / 2$ and $\pi / 2<\theta_{k} \leqslant \pi$ implies that the beam of Eq. (29) for a weighting function peaked about $\theta_{k}=0$ contains a large amount of forward propagation and a small amount of backward propagation, which is equivalent to the presence of a weak standing-wave component in addition to the dominant forward propagating wave. To more accurately model an experimental focused laser beam that is purely forward propagating, in this section 1 give a prescription for minimizing the standing-wave component by a suitable choice of the beam shape coefficients in the partial-wave decomposition of $A\left(\theta_{k}, \phi_{k}\right)$.

Since $A\left(\theta_{k}, \phi_{k}\right)$ is defined on the surface of a sphere in Fourier transform space, it can be expanded in terms of spherical harmonics,

$$
A\left(\theta_{k}, \phi_{k}\right)=\sum_{n, m} \alpha_{n m} Y_{n}^{m}\left(\theta_{k}, \phi_{k}\right)
$$

with $n \geqslant 0$ and $-n \leqslant m \leqslant n$, and

$$
\alpha_{n m}=\int_{0}^{\pi} \sin \theta_{k} \mathrm{~d} \theta_{k} \int_{0}^{2 \pi} \mathrm{d} \phi_{k} A\left(\theta_{k}, \phi_{k}\right) Y_{n}^{m *}\left(\theta_{k}, \phi_{k}\right) .
$$

Substituting Eq. (30) and the partial-wave expansion ${ }^{18}$ of $\exp (i \mathbf{k} \cdot \mathbf{r})$ into Eq. (29), one obtains the partial-wave expansion of $\psi_{\text {beam }}$,

$$
\psi_{\text {beam }}(r, \theta, \phi)=\sum_{n, m} i^{n} \alpha_{n m} j_{n}(k r) Y_{n}^{m}(\theta, \phi) .
$$

For the remainder of this section, I consider a circularly symmetric beam with $m=0$ so that the angular spectrum weighting factor is independent of $\phi_{k}$ and can be written simply as $A\left(\theta_{k}\right)$. Defining the beam shape coefficients $\alpha_{n}$ by

$$
\alpha_{n m}=[4 \pi(2 n+1)]^{1 / 2} \alpha_{n} \delta_{m, 0},
$$

Eq. (29) for the circularly symmetric beam simplifies to the familiar form,

$$
\psi_{\text {beam }}(r, \theta, \phi)=\sum_{n=0}^{\infty} i^{n}(2 n+1) \alpha_{n} j_{n}(k r) P_{n}(\cos \theta) .
$$

The angular spectrum weighting function $A\left(\theta_{k}\right)$ and the beam shape coefficients $\alpha_{n}$ are related by

$$
\begin{aligned}
A\left(\theta_{k}\right) & =\sum_{n=0}^{\infty}(2 n+1) \alpha_{n} P_{n}\left(\cos \theta_{k}\right) \\
\alpha_{n} & =\left(\frac{1}{2}\right) \int_{0}^{\pi} \sin \theta_{k} \mathrm{~d} \theta_{k} A\left(\theta_{k}\right) P_{n}\left(\cos \theta_{k}\right) .
\end{aligned}
$$

\section{B. Scattering of a Sphere by the Alternate Angular Spectrum Beam}

If the beam of Eq. (34) is incident on a sphere of radius $a$ and refractive index $N$, the partial-wave scattered and interior amplitudes defined in Eqs. (24) and (25) have the usual forms, 


$$
\begin{aligned}
& b_{n}=\alpha_{n} M_{n} /\left(M_{n}+i D_{n}\right), \\
& d_{n}=\alpha_{n}\left[-1 /(k a)^{2}\right] /\left(M_{n}+i D_{n}\right) .
\end{aligned}
$$

Thus the beam description of Eqs. (29) and (34) enjoys a far greater simplicity for partial-wave expansion and generalized Lorentz-Mie scattering calculations than did the beam description of Eq. (3). However, while all the nonzero beam shape coefficients $\alpha_{n}$ in Eq. (17b) were obtainable in terms of the beam amplitude $\psi_{\text {beam }}(\rho, 0)$, in the present situation they are not. Consider Eq. (34) for $\theta$ $=\pi / 2$. One has

$$
\psi_{\text {beam }}(\rho, 0)=\sum_{n \text { even }}(2 n+1)[(n-1) ! ! /(n) ! !] \alpha_{n} j_{n}(k r)
$$

Inverting Eq. (38) one obtains ${ }^{19}$

$$
\alpha_{n}=\int_{0}^{\infty} k \mathrm{~d} \rho j_{n}(k \rho) \psi_{\text {beam }}(\rho, 0) / \int_{0}^{\infty} k \mathrm{~d} \rho j_{n}(k \rho),
$$

for even $n$ only. This result is identical to that obtained for the odd partial waves in Eq. (17b). But the difference is that Eq. (39) relates only the even beam shape coefficients to the beam amplitude in the $z=0$ plane and gives no clue as to how to evaluate the beam shape coefficients for the odd partial waves. The reason for this is that Eq. (34) describes the forward-propagating beam as the sum of two standing waves, one corresponding to the even partial waves and the other corresponding to the odd partial waves. The same is true of Eq. (29), where the even and odd parts of $A\left(\theta_{k}\right)$ describe two standing waves that superpose to form the propagating wave. The even standing wave is nonzero in the $z=0$ plane and can thus be related to $\psi_{\text {beam }}(\rho, 0)$, whereas the odd standing wave vanishes in the $z=0$ plane. Though $\psi_{\text {beam }}(\rho, 0)$ provides no information on the value of the beam shape coefficients $\alpha_{n}$ for odd $n$, these coefficients are nonetheless required in order to construct the full beam via Eq. (34).

\section{Minimizing the Standing-Wave Content of the Alternate Angular Spectrum Beam} A procedure for determining the odd $n$ beam shape coefficients that minimizes the standing-wave portion of $\psi_{\text {beam }}$ is as follows. Equation (34) may be rewritten as

$$
\psi_{\text {beam }}(r, \theta, \phi)=\sum_{n=0}^{\infty} i^{n}(n+1 / 2) \alpha_{n}\left[h_{n}^{(1)}(k r)+h_{n}^{(2)}(k r)\right] P_{n}(\cos \theta) .
$$

On the positive $z$ axis far from the origin this becomes

$$
\begin{aligned}
\psi_{\text {beam }}(z) \rightarrow & (-i / k z) \sum_{n=0}^{\infty}(n+1 / 2) \alpha_{n}[\exp (\mathrm{i} k z) \\
& \left.-(-1)^{n} \exp (-\mathrm{i} k z)\right] .
\end{aligned}
$$

If the beam shape coefficients $\alpha_{n}$ satisfy

$$
\sum_{n \text { even }}(n+1 / 2) \alpha_{n}=\sum_{n \text { odd }}(n+1 / 2) \alpha_{n}
$$

the beam evaluated on the $z$ axis in the $z \rightarrow \infty$ limit becomes the pure traveling wave $\exp (\mathrm{i} k z)$. If a small part of the $\exp (-\mathrm{i} k z)$ beam had remained, it could have been added to an equal amount of $\exp (\mathrm{i} k z)$ beam to produce the standing-wave portion of the beam in addition to the dominant $\exp (\mathrm{i} k z)$ traveling wave. Equations (40) and (42) also reduce to $\exp (\mathrm{i} k z)$ on the $z$ axis far upstream on the beam in the $z \rightarrow-\infty$ limit. If the beam is not too tightly focused in the $z=0$ plane and many partial waves contribute to Eq. (34), a solution to Eq. (42) is

$$
\alpha_{n}=\frac{1}{2}\left(\alpha_{n+1}+\alpha_{n-1}\right)+\left[\frac{1}{2}\left(\alpha_{n+1}-\alpha_{n-1}\right) /\left(n+\frac{1}{2}\right)\right],
$$

i.e., each $\alpha_{n}$ for odd $n$ and $n \gg 1$ is approximately the average of the preceding even term $\alpha_{n-1}$ and the next even term $\alpha_{n+1}$. Qualitatively, Eq. (43) implies that the beam shape coefficients for odd $n$ smoothly interpolate between the values for the adjacient even $n$ coefficients. An easy way of accomplishing this is that if $\alpha_{n}$ for even $n$ can be written as a slowly varying function of the variable $n$, then $\alpha_{n}$ for odd $n$ is given by the same function. Thus as long as the beam is either loosely or moderately tightly focused in the $z=0$ plane so that many $\alpha_{n}$ contribute to the partial wave sum, the prescription

$$
\alpha_{n}=\int_{0}^{\infty} k \mathrm{~d} \rho j_{n}(k \rho) \psi_{\text {beam }}(\rho, 0) / \int_{0}^{\infty} k \mathrm{~d} \rho j_{n}(k \rho)
$$

for all $n$, substituted into Eq. (34), produces a beam $\psi_{\text {beam }}(r, \theta, \phi)$ for which the standing-wave portion is minimal. As an example of evaluating the beam shape coefficients using Eq. (44), the Gaussian beam profile in the $z$ $=0$ plane,

$$
\psi_{\text {beam }}(\rho, 0)=\exp \left(-\rho^{2} / w_{0}^{2}\right)
$$

gives rise to the beam shape coefficients, ${ }^{20}$

$$
\begin{aligned}
\alpha_{n}= & t_{n}\left[2^{(n+2) / 2}(n) ! !\right] M[(n+1) / 2, n+3 / 2, \\
& \left.-1 / 4 s^{2}\right] /\left[\pi^{1 / 2}(2 s)^{n+1}(2 n+1) ! !\right],
\end{aligned}
$$

where $M$ is the confluent hypergeometric function ${ }^{21}$ and

$$
t_{n}=\left\{\begin{array}{ll}
1, & \text { if } n=\text { even } \\
(2 \pi)^{1 / 2}, & \text { if } n=\text { odd }
\end{array} .\right.
$$

As another application of Eq. (44), the localized beam model has been used to approximate a focused Gaussian beam $^{22}$ and a top-hat beam ${ }^{23}$ and can be simply derived from Eq. (44) as follows. The spherical Bessel function $j_{n}(k \rho)$ reaches its first and largest relative maximum near

$$
k \rho \approx n+\frac{1}{2} \text {. }
$$

Replacing $j_{n}(k \rho)$ by a Dirac delta function at this location and performing the integration over $\rho$ in the numerator and denominator, one obtains

$$
\alpha_{n} \approx \psi_{\text {beam }}\left[\left(n+\frac{1}{2}\right) / k, 0\right],
$$

which is the general form for the localized model beam shape coefficients for an on-axis beam. ${ }^{22}$ Applying this prescription to the Gaussian profile of Eq. (45) gives

$$
\alpha_{n} \approx \exp \left[-s^{2}\left(n+\frac{1}{2}\right)^{2}\right]
$$

As crude as this approximation may be to the optimal beam shape coefficients of Eqs. (46a) and (46b), the local- 
ized model for a focused Gaussian beam in generalized Lorentz-Mie scattering calculations has given results that compare very favorably to experiments. ${ }^{24,25}$

\section{DISCUSSION}

Each of the two angular spectra of plane waves examined here is naturally suited to certain types of calculations and proves to be rather awkward in other situations. The angular spectrum examined in Section 2 reproduces the Rayleigh-Sommerfeld propagator of scalar diffraction theory, and as a result it is well suited to diffraction calculations. But the evanescent waves lead to an expansion in terms of partial waves where the beam consists of only odd partial waves. Scattering of such a beam by a spherical particle produces novel effects, such as the coupling of even scattered partial waves to the neighboring odd partial waves of the incident beam.

The alternate angular spectrum examined in Section 3 is naturally suited to partial-wave scattering calculations. But it possesses two complications of its own. First, since the angular spectrum includes plane waves propagating in both the positive and negative directions, it cannot exactly describe a purely forward-propagating experimental beam. A procedure is proposed here for minimizing the beam's standing-wave component that exploits the second complication, namely, that only the even partial-wave beam shape coefficients are related to the beam amplitude in its focal plane. As a result one is free to choose the odd partial-wave beam shape coefficients so as to minimize the unwanted character of the beam. This prescription is given by Eq. (44), which I consider to be the principal result of this paper, and can also be used to evaluate the on-axis beam shape coefficients $g_{n}$ for an electromagnetic beam for which the electric and magnetic field wave amplitudes are vectors. In the partial-wave analysis of a beam incident on a particle whose center is at the origin of coordinates, one commonly associates each partial wave with a narrow portion of the beam that propagates past the origin with the impact parameter $\rho$ as given by ${ }^{26}$ Eq. (47). As was seen in Section 3, weighting all portions of the wave propagating past the origin with different impact parameters with the spherical Bessel function $j_{n}(k \rho)$ gives a more correct prescription for identifying the portion of the impact parameter interval that corresponds to a given partial wave. The simple but experimentally accurate localized beam model of Eq. (48) is an approximation to this prescription.

From a practical point of view, the complications arising from the evanescent waves of the angular spectrum of Section 2 and the standing-wave portion of the angular spectrum of Section 3 are commonly ignored in the paraxial approximation, which describes a weakly focused beam. If the weighting functions $A\left(k_{x}, k_{y}\right)$ in Eq. (3) and $A\left(\theta_{k}, \phi_{k}\right)$ in Eq. (29) are sharply peaked about $k_{x}=k_{y}=0$ and $\theta_{k}=0$, respectively, both the evanescent and backward propagating portions of the angular spectra are negligible and can be ignored. It is only in the case of moderately tight focusing when the sharp peaking approximation is no longer quantitatively accurate that the differing consequences of the two angular spectra become apparent.

\section{ACKNOWLEDGMENT}

This research was supported in part by the Subsonic Fixed Wing Program at the NASA-Glenn Research Center under grant NAG3-2774.

J. A. Lock's e-mail address is j.lock@csuohio.edu.

\section{REFERENCES}

1. J. W. Goodman, Introduction to Fourier Optics (McGrawHill, 1968), pp. 48-51.

2. M. Born and E. Wolf, Principles of Optics, 6th ed. (Cambridge U. Press, 1980), pp. 561-564.

3. C. Yeh, S. Colak, and P. Barber, "Scattering of sharply focused beams by arbitrarily shaped dielectric particles: an exact solution," Appl. Opt. 21, 4426-4433 (1982).

4. H. C. van de Hulst, Light Scattering by Small Particles (Dover, 1981), p. 121.

5. J. P. Barton, D. R. Alexander, and S. A. Schaub, "Internal and near-surface electromagnetic fields for a spherical particle irradiated by a focused laser beam,” J. Appl. Phys. 64, 1632-1639 (1988)

6. G. Gouesbet, B. Maheu, and G. Grehan, "Light scattering from a sphere arbitrarily located in a Gaussian beam, using a Bromwich formulation," J. Opt. Soc. Am. A 5, 1427-1443 (1988).

7. H. C. van de Hulst, Light Scattering by Small Particles (Dover, 1981), pp. 122-125.

8. D. J. Griffiths, Introduction to Quantum Mechanics, 2nd ed. (Pearson Prentice Hall, 2005), p. 418.

9. K. Gottfried and T.-M. Yan, Quantum Mechanics: Fundamentals, 2nd ed. (Springer, 2003), pp. 84-85, Eqs. (354) and (355).

10. I. S. Gradshteyn and I. M. Ryzhik, Table of Integrals, Series, and Products (Academic, 1965), p. 761, Eq. (6.738.1).

11. J. W. Goodman, Introduction to Fourier Optics (McGrawHill, 1968), pp. 43-45.

12. O. R. Cruzan, "Translational addition theorems for spherical vector wave functions," Q. Appl. Math. 20, 33-40 (1962), App. B, Eqs. (B.1) and (B.2).

13. A. Messiah, Quantum Mechanics (Wiley, 1966), Vol. 1, p. 497, Eqs. (B.99)-(B.101).

14. Ref. 10, p. 684, Eq. (6.561.14).

15. G. Arfken, Mathematical Methods for Physicists, 3rd ed. (Academic, 1965), p. 545, Eq. (10.33b).

16. A. Messiah, Quantum Mechanics (Wiley, 1966), Vol. 2, pp. 1057-1059, Eqs. (C.16), (C.22), (C.23a), and (C.23b).

17. J. A. Lock, "Excitation of morphology-dependent resonances and van de Hulst's localization principle," Opt. Lett. 24, 427-429 (1999).

18. A. Messiah, Quantum Mechanics (Wiley, 1966), Vol. 1, p. 497, Eq. (B105).

19. G. Arfken, Mathematical Methods for Physicists, 3rd ed. (Academic, 1965), pp. 629-630, Eqs. (11.174) and (11.175).

20. I. S. Gradshteyn and I. M. Ryzhik, Table of Integrals, Series, and Products (Academic, 1965), p. 716, Eq. (6.631.1).

21. G. Arfken, Mathematical Methods for Physicists, 3rd ed. (Academic, 1965), p. 753, Eq. (13.134).

22. B. Maheu, G. Grehan, and G. Gousebet, "Ray localization in Gaussian beams," Opt. Commun. 70, 259-262 (1989).

23. G. Gouesbet, J. A. Lock, and G. Grehan, "Partial-wave representations of laser beams for use in light-scattering calculations," Appl. Opt. 34, 2133-2143 (1995).

24. J. A. Lock and J. T. Hodges, "Far-field scattering of an axisymmetric laser beam of arbitrary profile by an on-axis spherical particle,” Appl. Opt. 35, 4283-4290 (1996).

25. J. A. Lock and J. T. Hodges, "Far-field scattering of a non-Gaussian off-axis axisymmetric laser beam by a spherical particle," Appl. Opt. 35, 6605-6616 (1996).

26. H. C. van de Hulst, Light Scattering by Small Particles (Dover, 1981), pp. 208-209. 